\title{
An animal experimental study of porous magnesium scaffold degradation and osteogenesis
}

\author{
Y.J. Liu' ${ }^{1}$, Z.Y. Yang ${ }^{1}$, L.L. Tan ${ }^{2}$, H. Li ${ }^{1}$ and Y.Z. Zhang ${ }^{1,3}$ \\ ${ }^{1}$ The Third Hospital of Hebei Medical University, Shijiazhuang, China \\ ${ }^{2}$ Institute of Metal Research, Chinese Academy of Sciences, Shenyang, China \\ ${ }^{3}$ The Key Orthopedic Biomechanics Laboratory of Hebei Province, Shijiazhuang, China
}

\begin{abstract}
Our objective was to observe the biodegradable and osteogenic properties of magnesium scaffolding under in vivo conditions. Twelve 6-month-old male New Zealand white rabbits were randomly divided into two groups. The chosen operation site was the femoral condyle on the right side. The experimental group was implanted with porous magnesium scaffolds, while the control group was implanted with hydroxyapatite scaffolds. X-ray and blood tests, which included serum magnesium, alanine aminotransferase (ALT), creatinine (CREA), and blood urea nitrogen (BUN) were performed serially at 1,2 , and 3 weeks, and 1,2 , and 3 months. All rabbits were killed 3 months postoperatively, and the heart, kidney, spleen, and liver were analyzed with hematoxylin and eosin (HE) staining. The bone samples were subjected to microcomputed tomography scanning (micro-CT) and hard tissue biopsy. SPSS 13.0 (USA) was used for data analysis, and values of $\mathrm{P}<0.05$ were considered to be significant. Bubbles appeared in the X-ray of the experimental group after 2 weeks, whereas there was no gas in the control group. There were no statistical differences for the serum magnesium concentrations, ALT, BUN, and CREA between the two groups $(P>0.05)$. All HE-stained slices were normal, which suggested good biocompatibility of the scaffold. Micro-CT showed that magnesium scaffolds degraded mainly from the outside to inside, and new bone was ingrown following the degradation of magnesium scaffolds. The hydroxyapatite scaffold was not degraded and had fewer osteoblasts scattered on its surface. There was a significant difference in the new bone formation and scaffold bioabsorption between the two groups $(9.29 \pm 1.27$ vs $1.40 \pm 0.49$ and $7.80 \pm 0.50$ vs $0.00 \pm 0.00 \mathrm{~mm}^{3}$, respectively; $\mathrm{P}<0.05$ ). The magnesium scaffold performed well in degradation and osteogenesis, and is a promising material for orthopedics.
\end{abstract}

Key words: Biodegradable; Osteogenic; Magnesium scaffold

\section{Introduction}

Stainless steel, pure titanium and its alloys, cobaltchromium-based alloys, etc., are commonly used metal materials for fixing bone fractures (1). As nonbiodegradable materials, they need a second surgery for removal after fracture healing, which not only increases the suffering of the patients, but also results in an increase in health care costs. To solve this problem, biodegradable metallic implants have been investigated $(1,2)$.

As a degradable metallic material, magnesium and its alloys have the following shortcomings: a rapid degradation rate and the release of hydrogen gas during degradation (3). A variety of modification methods such as alloying and surface treatments (microarc oxidation, ion implantation, plasma anodization, electro-deposition, etc.) have enhanced the corrosion resistance of magnesium and reduced the production of hydrogen considerably (3-5). However, current research has shown that the mechanical integrity of the magnesium alloy is maintained for no more than 12 weeks during the corrosion process, which means that plates, screws, and pins made of magnesium cannot be used to secure serious fractures. Until now, it has only had potential as a degradable orthopedic implant for clinical applications.

Therefore, in this study, we designed a porous magnesium scaffold, which was used to promote osteogenesis rather than load bearing, and the aim of the study was to observe the biodegradable and osteogenic properties of the magnesium scaffold under in vivo conditions.

Correspondence: Y.Z. Zhang, Department of Traumatic Center, The Third Hospital of Hebei Medical University, No. 139, the Zi Qiang Road, Shijiazhuang 050051, Hebei, China. Fax: +86-0311-8702-3626. E-mail: yzlingliu@126.com 


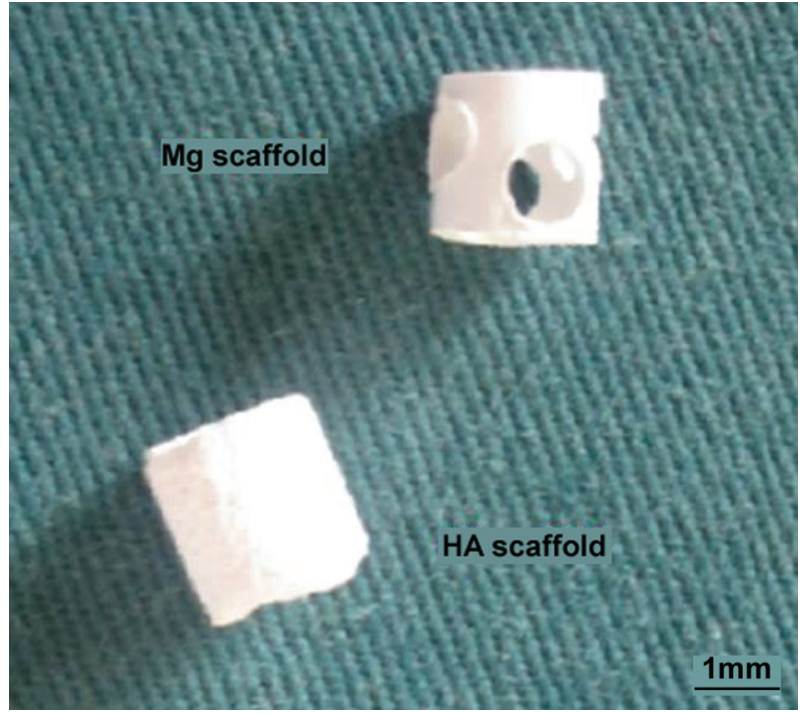

Figure 1. Example of the magnesium scaffold (top right) and hydroxyapatite scaffold (bottom left).

\section{Material and Methods}

\section{Sample preparation}

Pure magnesium (99.95\%) was cut from an extruded bar and then machined into hollow cylindrical scaffolds with dimensions of $5 \mathrm{~mm}$ in length, $6 \mathrm{~mm}$ outside diameter, $4 \mathrm{~mm}$ inside diameter, and with four holes (1 $\mathrm{mm}$ in diameter) dispersed on the walls of the scaffold. The surface of the scaffold was modified by microarc oxidation technology and then sterilized with ethylene oxide. Six hydroxyapatite (HA) scaffolds with the same size as the magnesium scaffolds were implanted as controls. The magnesium and HA scaffolds are shown in Figure 1. Twelve male 6-month-old New Zealand white rabbits were purchased from the Experimental Animal Center of Hebei Medical University (batch number \#SCXK2008-003; China). All experimental procedures were approved by the Institutional Animal Care and Use Committee. The rabbits were randomly divided into two groups, the right femoral condyles of which were implanted with porous magnesium scaffolds and hydroxyapatite scaffolds, respectively.

\section{Surgical procedures}

All rabbits were anesthetized with a $1.0 \%$ pentobarbital sodium solution $(30 \mathrm{mg} / \mathrm{kg}$ ) by ear vein injection followed by $1 \%$ povidone-iodine disinfection and draping. A $1.0-\mathrm{cm}$ longitudinal incision was made laterally through the right knee. Muscle tissue was retracted to expose the femoral lateral condyle. A hand drill was used to drill a hole $5 \mathrm{~mm}$ in depth, with a diameter of $6 \mathrm{~mm}$. Subsequently, the scaffold was implanted into the prepared hole, and the wound was sutured layer-by-layer and covered with sterile dressing. After the operation, all rabbits received subcutaneous injections of $1 \mathrm{mg} / \mathrm{kg}$ gentamicin.

\section{Imaging studies}

X-ray radiography was conducted on the right femoral condyles of the rabbits in order to monitor the scaffold degradation, new bone formation, and gas production preoperation and 1, 2, 3, 4, 8, and 12 weeks post-operation.

\section{Blood tests}

Blood samples were taken from the rabbits, preoperation and 1, 2, 3, 4, 8, and 12 weeks postoperation. Tests for serum magnesium, alanine aminotransferase $(A L T)$, creatinine (CREA), and blood urea nitrogen (BUN) were conducted on an Olympus AU-1000 automatic biochemical analyzer (Japan).

\section{Histological examination}

All rabbits were killed 3 months post-operation. The heart, kidney, spleen, and liver tissues from the rabbits were analyzed with hematoxylin and eosin (HE) staining to verify whether degradation of the magnesium scaffold harmed these important visceral organs. Bone samples with implants were harvested and fixed in $10 \%$ buffered formalin for 3 days and then dehydrated in graded ethanol solution from $70 \%$ to $100 \%$ by immersing in each of the solutions for 3 days. Finally the bone samples were embedded in methyl methacrylate (Technovit 9100 New, Heraeus Kulzer, Germany) as per the manufacturer's instructions. Then all samples were scanned in a microcomputed tomography (micro-CT) device (Skyscan 1172, Skyscan Co., Germany) to investigate the degradation of the samples, as well as the new bone growth. After scanning, three-dimensional models were generated by CTVol (Skyscan Co.). The scaffold degradation rate, which was calculated as original implant volume minus residual implant volume divided by original implant volume, and the new bone growth volume were then analyzed using a CTAn program (Skyscan Co.), which is used to examine (micro-CT) datasets for morphometry and densitometry. The embedded samples were then cut into sections with a thickness of $50-70 \mu \mathrm{m}$ and then stained with Giemsa methylene blue-magenta stain. Morphological and histological analyses were performed under a light microscope to observe bone growth and integration with the host tissue.

The SPSS statistical software (Version 13.0) was used for data analysis. Probability values less than 0.05 were considered significant.

\section{Results}

\section{Imaging results}

Bubbles appeared in the X-rays of the New Zealand white rabbits in the experimental group after 2 weeks, and 


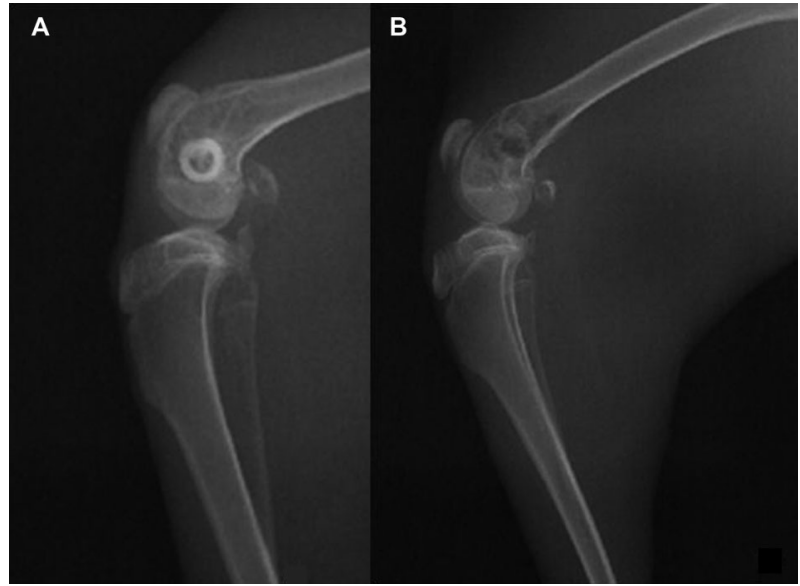

Figure 2. There was no sign of gas formation in the X-ray of New Zealand rabbit knee with the HA scaffold 2 weeks after implantation $(A)$. There were signs of gas formation in the $\mathrm{x}$-ray of New Zealand rabbit knee with the Mg scaffold 2 weeks after implantation $(B)$.

disappeared 2 months later, but there was no gas formation in the control group (Figure 2).

The micro-CT scans showed (Figure 3) that the magnesium scaffolds degraded mainly from the outside to inside and the new bone was ingrown following degradation of the magnesium scaffold. There was little degradation of the magnesium scaffold. The new bone formation was mainly from inside to outside, with an average volume of new bone growth of $9.29 \pm 1.27 \mathrm{~mm}^{3}$ and degradation volume of $7.80 \pm 0.50 \mathrm{~mm}^{3}$, which was almost one-third of the total volume of the scaffold. The hydroxyapatite scaffold was not degraded, with a degradation volume of $0 \mathrm{~mm}^{3}$, and there were fewer osteoblasts scattered on its surface, with an average volume of new bone growth of $1.40 \pm 0.49 \mathrm{~mm}^{3}$. There was a significant difference in new bone formation and scaffold bioabsorption between the two groups $(\mathrm{P}<0.05$; Table 1).
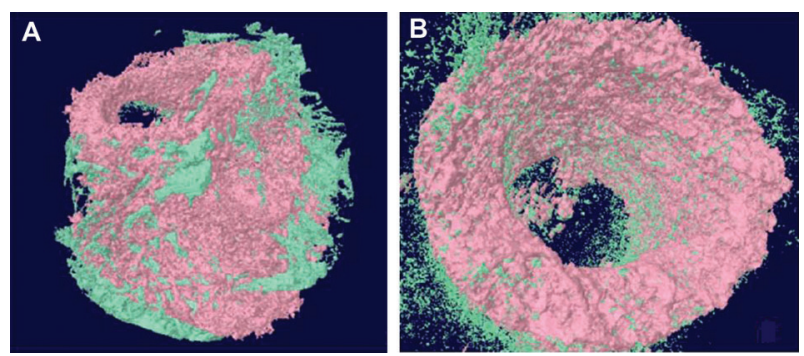

Figure 3. Micro-CT image of the magnesium scaffold: the new bone tissue in-growth 3 months post-operation $(A)$. Micro-CT image of hydroxyapatite scaffold: visible new bone scattered on the surface of hydroxyapatite 3 months post-operation $(B)$ (green indicates newly generated bone tissue).
Table 1. Volume of new bone formation and of scaffold bioabsorption for the magnesium scaffold experimental group and hydroxyapatite scaffold control group $\left(\mathrm{mm}^{3}\right)$.

\begin{tabular}{llcc}
\hline & Mean \pm SD & $t$ & $\mathrm{P}$ \\
\hline $\begin{array}{l}\text { New bone formation } \\
\quad \text { Experimental group }\end{array}$ & $9.29 \pm 1.27$ & 14.214 & 0.000 \\
$\quad$ Control group & $1.40 \pm 0.49$ & & \\
Scaffold bioabsorption & & & \\
$\quad$ Experimental group & $7.80 \pm 0.50$ & 37.910 & 0.000 \\
$\quad$ Control group & $0.00 \pm 0.00$ & & \\
\hline
\end{tabular}

The $t$-test was used for statistical analyses.

\section{Hematology results}

There were no statistical differences in the serum magnesium concentrations, ALT, and CREA, and BUN between the experimental group and control group $(P>0.05$; Table 2), which suggested that the magnesium scaffold had good biocompatibility in vivo.

\section{Results of histological examination}

All HE-stained slices of heart, liver, kidney, and spleen were normal, which suggested good biocompatibility of the magnesium scaffold in the hard tissue biopsy (Figure 4).

There was more new bone formation in the magnesium group than the hydroxyapatite group in the hard tissue biopsies (Figure 5).

\section{Discussion}

The main purpose of this study was to use a porous magnesium scaffold to promote bone formation, and the above results indicate that this was accomplished. McBride (6) and Verbrugge (7) completed a series of studies on the clinical feasibility of biodegradable magnesium material during the 1930s. They found that magnesium had the advantages of being biodegradable and having osteoblasts scattered on its surface, which gave magnesium the potential to become a novel biodegradable orthopedic internal fixation material, but its rapid degradation rate and the fact that it produced large amounts of hydrogen in the process of rapid degradation were the biggest obstacles, which prevented its clinical application (8-13).

Since the beginning of the present century, surface modification and alloy technology have been used to treat magnesium, aimed at slowing its degradation rate, so that the small amounts of hydrogen generated in the process of slow degradation could be absorbed by the body. Given that small amounts of hydrogen are conducive to health, these treatments allow magnesium to be used as an orthopedic fixation material with a fixed expiration date (14-19). Nevertheless, the degradation rate of material made from magnesium is still too fast, and it cannot meet the general requirements of clinical orthopedic internal fixation devices. Therefore, in this study, we avoided the 
Table 2. Concentrations of serum magnesium, creatinine, ALT, and BUN of the magnesium scaffold group and the hydroxyapatite scaffold control group (mM) at baseline to 12 weeks.

\begin{tabular}{|c|c|c|c|c|c|c|c|}
\hline & 0 weeks & 1 week & 2 weeks & 3 weeks & 4 weeks & 8 weeks & 12 weeks \\
\hline \multicolumn{8}{|c|}{ Magnesium (mM) } \\
\hline MG & $1.015 \pm 0.094$ & $1.145 \pm 0.092$ & $1.165 \pm 0.071$ & $1.003 \pm 0.102$ & $1.025 \pm 0.182$ & $1.018 \pm 0.142$ & $1.003 \pm 0.106$ \\
\hline $\mathrm{HA}$ & $1.020 \pm 0.076$ & $1.135 \pm 0.149$ & $1.045 \pm 0.137$ & $1.065 \pm 0.076$ & $1.065 \pm 0.082$ & $1.048 \pm 0.074$ & $1.033 \pm 0.057$ \\
\hline$t$ & -0.100 & 0.140 & 1.905 & -1.183 & -0.491 & -0.460 & -0.610 \\
\hline $\mathrm{P}$ & 0.922 & 0.892 & 0.086 & 0.264 & 0.634 & 0.656 & 0.556 \\
\hline \multicolumn{8}{|c|}{ Creatinine $(\mu \mathrm{M})$} \\
\hline $\mathrm{Mg}$ & $73.83 \pm 10.80$ & $76.67 \pm 17.25$ & $76.83 \pm 15.29$ & $72.83 \pm 8.08$ & $71.50 \pm 8.53$ & $72.00 \pm 5.93$ & $71.67 \pm 5.54$ \\
\hline $\mathrm{HA}$ & $70.17 \pm 10.01$ & $69.83 \pm 7.41$ & $70.67 \pm 5.96$ & $70.67 \pm 4.59$ & $70.83 \pm 3.82$ & $71.83 \pm 5.12$ & $71.83 \pm 3.06$ \\
\hline$t$ & 0.610 & 0.892 & 0.921 & 0.571 & 0.175 & 0.052 & -0.065 \\
\hline $\mathrm{P}$ & 0.555 & 0.394 & 0.379 & 0.581 & 0.865 & 0.959 & 0.950 \\
\hline \multicolumn{8}{|c|}{ BUN (mM) } \\
\hline $\mathrm{Mg}$ & $2.33 \pm 1.15$ & $2.26 \pm 0.78$ & $2.26 \pm 0.69$ & $2.28 \pm 0.66$ & $2.29 \pm 0.65$ & $2.27 \pm 0.65$ & $2.25 \pm 0.64$ \\
\hline $\mathrm{HA}$ & $2.47 \pm 1.12$ & $2.36 \pm 0.80$ & $2.35 \pm 0.80$ & $2.34 \pm 0.76$ & $2.31 \pm 0.78$ & $2.32 \pm 0.78$ & $2.34 \pm 0.78$ \\
\hline$t$ & -0.211 & -0.223 & -0.212 & -0.133 & -0.056 & -0.121 & -0.198 \\
\hline $\mathrm{P}$ & 0.837 & 0.828 & 0.836 & 0.896 & 0.956 & 0.906 & 0.847 \\
\hline \multicolumn{8}{|c|}{$\operatorname{ALT}(\mu \mathrm{M})$} \\
\hline $\mathrm{Mg}$ & $40.33 \pm 7.42$ & $43.83 \pm 5.67$ & $46.67 \pm 4.89$ & $45.67 \pm 4.32$ & $45.00 \pm 4.69$ & $44.17 \pm 5.64$ & $44.50 \pm 4.81$ \\
\hline $\mathrm{HA}$ & $43.17 \pm 4.07$ & $48.83 \pm 2.32$ & $52.00 \pm 2.28$ & $52.83 \pm 4.49$ & $50.83 \pm 4.54$ & $48.83 \pm 2.99$ & $48.67 \pm 1.03$ \\
\hline$t$ & -0.820 & -1.999 & -2.423 & -2.817 & -2.190 & -1.791 & -2.076 \\
\hline $\mathrm{P}$ & 0.431 & 0.073 & 0.056 & 0.058 & 0.053 & 0.104 & 0.065 \\
\hline
\end{tabular}

MG: magnesium scaffold; HA: hydroxyapatite scaffold; BUN: blood urea nitrogen; ALT: alanine aminotransferase. The $t$-test was used for statistical analyses and no significant differences were found between groups or times $(P>0.05)$.

difficulties associated with internal fixation devices and used its biodegradable and osteogenic properties as a starting point, by trying to develop biodegradable scaffolds implanted to promote osteogenesis in the body.

Hydroxyapatite has served as an alternative to autogenous grafts, but questions regarding its biocompatibility, the risk of infection, and its slow set times have hampered its acceptance. The main ingredient of hydroxyapatite is calcium phosphate. It is similar to the inorganic constituents of human bone in chemical composition and physical properties and is one of the most mature orthopedic biomaterials used to promote osteogenesis; therefore, it
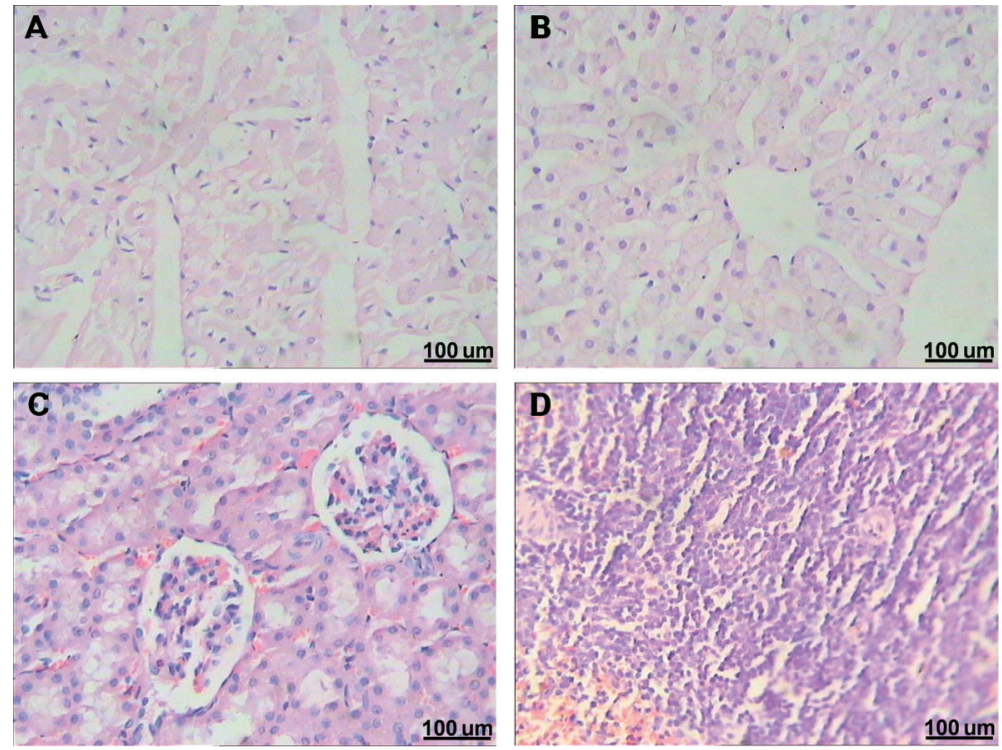

Figure 4. Hematoxylin-eosin staining of the heart $(A)$, liver $(B)$, kidney $(C)$, and spleen $(D)$ of the rabbit with magnesium scaffold 3 months after implantation showed no pathological changes $(40 \times)$. 


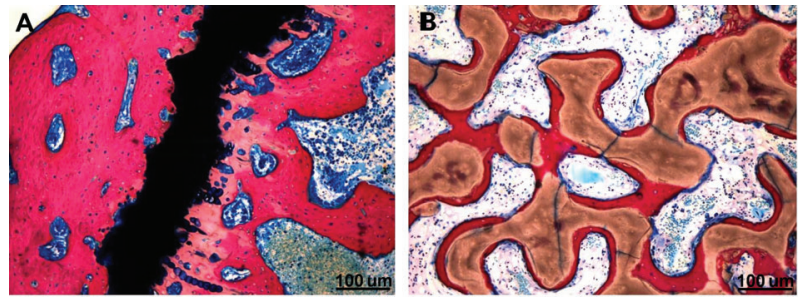

Figure 5. There was obvious new bone formation in the hardtissue sections of the magnesium scaffold and surrounding tissue 3 months post-operation $(A)$. There was no obvious new bone formation in the hard-tissue sections of the hydroxyapatite scaffold and surrounding tissue 3 months post-operation $(B)$. Methylene blue-magenta staining $(100 \times)$.

was used as a control in the experiments. However, its degradation rate and osteogenic performance are deficient, and it is expensive (20-23).

The porous magnesium scaffolds exhibited good degradation and osteogenesis in contrast to the hydroxyapatite scaffolds in this experiment. There were no pathological changes of the heart, liver, spleen, and kidneys of the animals of the experimental group, and biochemical indicators of tests showed normal liver and kidney function, which indicated that the porous magnesium scaffolds had good biological compatibility. However, a certain level of hydrogen produced mild swelling in the knees of rabbits in the experimental group 2 weeks later, which did not return to normal until 2 months later. This indicated that the thickness of the protective magnesium oxide produced by microarc oxidation technology was inadequate. Therefore, in future studies, improvements in the microarc oxidation process should be made, or a polylactic acid coating should be applied to the surface of the magnesium scaffolds so that, as the magnesium is degraded, the hydrogen gas produced can be absorbed by the body and swelling at the knee can be prevented.

Recent studies (24-27) suggest that the first reaction of magnesium in the body is oxidation $\left(\mathrm{Mg} \rightarrow \mathrm{Mg}^{2+}+2 \mathrm{e}\right)$; a reduction reaction then occurs $\left(2 \mathrm{H}_{2} \mathrm{O}+2 \mathrm{e} \rightarrow \mathrm{H}_{2}+2 \mathrm{OH}^{-}\right)$, generating hydroxide ions and magnesium ions, which combine to generate magnesium hydroxide as follows: $\mathrm{Mg}^{2+}+2 \mathrm{OH}^{-} \rightarrow \mathrm{Mg}(\mathrm{OH})_{2}$. Magnesium hydroxide is only slightly soluble in water, but the existence of a large number

\section{References}

1. Staiger MP, Pietak AM, Huadmai J, Dias G. Magnesium and its alloys as orthopedic biomaterials: a review. Biomaterials 2006; 27: 1728-1734, doi: 10.1016/j.biomaterials.2005. 10.003.

2. Xu L, Yu G, Zhang E, Pan F, Yang K. In vivo corrosion behavior of $\mathrm{Mg}-\mathrm{Mn}-\mathrm{Zn}$ alloy for bone implant application. J Biomed Mater Res A 2007; 83: 703-711, doi: 10.1002/ jbm.a.31273.

3. Gray JE, Luan B. Protective coatings on magnesium and its of chlorine ions in tissue fluid converts the slightly watersoluble magnesium hydroxide into a completely watersoluble magnesium chloride. This allows the hydroxide ion in tissue fluid to be in relative excess, resulting in alkaline tissue fluid. However, in the complex chemical environment of body tissue fluid, there also exist calcium ions, phosphate ions, etc.; thus, ultimately, $\mathrm{Mg}_{\mathrm{x}} \mathrm{Ca}_{\mathrm{y}}(\mathrm{PO} 4)_{\mathrm{z}}(\mathrm{OH})_{\mathrm{n}}$ complex compounds may be formed. Therefore, degradation of magnesium could promote deposition of calcium, which may be the reason magnesium degradation promotes osteoblast growth (28-30). It is consistent with the results we observed from micro-CT: that whenever magnesium degradation occurs in layers, new bone tissue forms sheets inside the magnesium scaffold layers. In addition, recent basic and clinical research has found that the right amount of hydrogen plays a positive role in maintaining normal function of the central nervous system, respiratory system, digestive system, etc. This would suggest that the final successful development of porous magnesium scaffolds may be able to provide beneficial effects of hydrogen on the human body, in addition to its performance through degradation and osteogenesis.

Shortcomings and deficiencies of this experiment are as follows. Because hydroxyapatite itself is brittle and the production process has constraints, hydroxyapatite scaffolds cannot be made into the same shapes as porous magnesium scaffolds, and this inevitably affects the experimental results. Another shortcoming was the implantation time, which was too short. However, this was only a preliminary study, and further research is needed that uses larger animals (e.g., sheep, Beagle dogs) using magnesium and hydroxyapatite scaffolds with exactly the same shape and extending the time of implantation.

These experiments are a preliminary confirmation that magnesium scaffolds perform well with respect to degradation and osteogenesis and that they are a promising biodegradable material for promoting osteogenesis, but not for internal fixation.

\section{Acknowledgments}

Research supported mainly by the Hebei Province Government (\#12966116D). alloys - a critical review. J Alloys Compd 2002; 336: 88-113 doi: 10.1016/S0925-8388(01)01899-0.

4. Denkena B, Witte F, Podolsky C, Lucas A. Degradable implants made of magnesium alloys. Proceedings of the 5th Euspen International Conference, Montpellier, France. Montpellier: 2005.

5. Kaesel VT, Bach PT, Haferkamp H, Witte F, Windhagen H. Approach to control the corrosion of magnesium by alloying Proceedings of the Sixth International Conference on 
Magnesium Alloys and their Applications. New York: WileyVch; 2004. p. 534-539.

6. McBride E. Absorbable metal in bone surgery. JAMA 1938; 111: 2464-2467, doi: 10.1001/jama.1938.02790530018007.

7. Verbrugge J. Le material metallique resorbable en chirurgie osseuse. Presse Med 1934; 23: 460-465.

8. Nakao A, Sugimoto R, Billiar TR, McCurry KR. Therapeutic antioxidant medical gas. J Clin Biochem Nutr 2009; 44: 113, doi: 10.3164/jcbn.08-193R.

9. Chen F, Zhou H, Yao B, Qin Z, Zhang Q. Corrosion resistance property of the ceramic coating obtained through microarc oxidation on the AZ31 magnesium alloy surfaces. Surf Coat Technol 2007; 201: 4905-4908, doi: 10.1016/j. surfcoat.2006.07.079.

10. Wang $Y Q$, Wu $K$, Zheng MY. Effects of reinforcement phases in magnesium matrix composites on microarc discharge behavior and characteristics of microarc oxidation coatings. Surf Coat Technol 2006; 201: 353-360, doi: 10.1016/j.surfcoat.2005.11.129.

11. Bakkar A, Neubert V. Improving corrosion resistance of magnesium-based alloys by surface modification with hydrogen by electrochemical ion reduction (EIR) and by plasma immersion ion implantation (PIII). Corr Sci 2005; 47: 1211-1225, doi: 10.1016/j.corsci.2004.06.027.

12. Li GY, Lian JS, Niu LY, Jiang ZH, Jiang Q. Growth of zinc phosphate coatings on AZ91D magnesium alloy. Surf Coat Technol 2006; 201: 1814-1820, doi: 10.1016/j.surfcoat. 2006.03.006.

13. Zhao $M$, Wu S, An $P$, Luo J, Fukuda $Y$, Nakae $H$. Microstructure and corrosion resistance of a chromium-free multi-elements complex coating on AZ91D magnesium alloy. Mater Chem Phys 2006; 99: 54-60, doi: 10.1016/j. matchemphys.2005.08.078.

14. Huang CS, Kawamura T, Toyoda Y, Nakao A. Recent advances in hydrogen research as a therapeutic medical gas. Free Radic Res 2010; 44: 971-982, doi: 10.3109/10715762. 2010.500328.

15. Xie K, Yu Y, Pei Y, Hou L, Chen S, Xiong L, et al. Protective effects of hydrogen gas on murine polymicrobial sepsis via reducing oxidative stress and HMGB1 release. Shock 2010; 34: 90-97, doi: 10.1097/SHK.0b013e3181cdc4ae.

16. Xu L, Yamamoto A. Characteristics and cytocompatibility of biodegradable polymer film on magnesium by spin coating. Colloids Surf B Biointerfaces 2012; 93: 67-74, doi: 10.1016/ j.colsurfb.2011.12.009.

17. Waizy $\mathrm{H}$, Weizbauer $\mathrm{A}$, Maibaum $\mathrm{M}$, Witte $\mathrm{F}$, Windhagen $\mathrm{H}$, Lucas A, et al. Biomechanical characterisation of a degradable magnesium-based (MgCa0.8) screw. J Mater Sci Mater Med 2012; 23: 649-655, doi: 10.1007/s10856011-4544-8.

18. Huan Z, Leeflang S, Zhou J, Zhai W, Chang J, Duszczyk J. In vitro degradation behavior and bioactivity of magnesiumBioglass ${ }^{\circledR}$ composites for orthopedic applications. J Biomed
Mater Res B Appl Biomater 2012; 100B: 437-446, doi: 10.1002/jbm.b.31968.

19. Choudhary L, Raman RK. Magnesium alloys as body implants: fracture mechanism under dynamic and static loadings in a physiological environment. Acta Biomater 2012; 8: 916-923, doi: 10.1016/j.actbio.2011.10.031.

20. Pataquiva-Mateus $\mathrm{AY}, \mathrm{Wu} \mathrm{HC}$, Lucchesi C, Ferraz MP, Monteiro FJ, Spector M. Supplementation of collagen scaffolds with SPARC to facilitate mineralization. $J$ Biomed Mater Res B Appl Biomater 2012; 100: 862-870, doi: $10.1002 / j \mathrm{jm} . b .32650$.

21. Nakamura M, Soya $T$, Hiratai $R$, Nagai $A$, Hashimoto $K$, Morita I, et al. Endothelial cell migration and morphogenesis on silk fibroin scaffolds containing hydroxyapatite electret. $J$ Biomed Mater Res A 2012; 100: 969-977, doi: 10.1002/jbm. a.34046.

22. Wang S, Wen S, Shen M, Guo R, Cao X, Wang J, et al. Aminopropyltriethoxysilane-mediated surface functionalization of hydroxyapatite nanoparticles: synthesis, characterization, and in vitro toxicity assay. Int J Nanomedicine 2011; 6: 3449-3459.

23. Tansavatdi K, Mangat DS. Calcium hydroxyapatite fillers. Facial Plast Surg 2011; 27: 510-516, doi: 10.1055/s-00311298783

24. Kraus T, Fischerauer SF, Hanzi AC, Uggowitzer PJ, Loffler JF, Weinberg AM. Magnesium alloys for temporary implants in osteosynthesis: in vivo studies of their degradation and interaction with bone. Acta Biomater 2012; 8: 1230-1238, doi: 10.1016/j.actbio.2011.11.008.

25. Gu XN, Li N, Zhou WR, Zheng YF, Zhao X, Cai QZ, et al Corrosion resistance and surface biocompatibility of a microarc oxidation coating on a $\mathrm{Mg}-\mathrm{Ca}$ alloy. Acta Biomater 2011; 7: 1880-1889, doi: 10.1016/j.actbio.2010. 11.034.

26. Wong HM, Yeung KW, Lam KO, Tam V, Chu PK, Luk KD, et al. A biodegradable polymer-based coating to control the performance of magnesium alloy orthopaedic implants. Biomaterials 2010; 31: 2084-2096, doi: 10.1016/j.biomaterials. 2009.11.111.

27. Gu XN, Zheng YF, Chen LJ. Influence of artificial biological fluid composition on the biocorrosion of potential orthopedic Mg-Ca, AZ31, AZ91 alloys. Biomed Mater 2009; 4: 065011, doi: 10.1088/1748-6041/4/6/065011.

28. Salahshoor M, Guo YB. Surface integrity of biodegradable Magnesium-Calcium orthopedic implant by burnishing. $J$ Mech Behav Biomed Mater 2011; 4: 1888-1904, doi: 10.1016/j.jmbbm.2011.06.006.

29. Chen D, He $Y$, Tao $H$, Zhang $Y$, Jiang $Y$, Zhang $X$, et al. Biocompatibility of magnesium-zinc alloy in biodegradable orthopedic implants. Int J Mol Med 2011; 28: 343-348.

30. Witte $F$. The history of biodegradable magnesium implants: a review. Acta Biomater 2010; 6: 1680-1692, doi: 10.1016/ j.actbio.2010.02.028. 